\title{
Lepton universality, flavour and number violation in $B$-meson and $\tau$-lepton decays at the $B$-factories
}

\section{Fergus WILSON*i}

STFC Rutherford Appleton Laboratory, Harwell Campus, Didcot, Oxon, OX11 OQX, UK

E-mail: Fergus.Wilsonestfc.ac.uk

I report on searches for evidence of the violation of Baryon Number, Lepton Number, Lepton Flavour and Lepton Universality in $\Upsilon$-mesons, $B$-mesons and $\tau$-lepton decays from the BABAR and Belle experiments.

9th International Workshop on the CKM Unitarity Triangle

28 November - 3 December 2016

Tata Institute for Fundamental Research (TIFR), Mumbai, India

* Speaker.

${ }^{\dagger}$ on behalf of the BABAR and Belle Collaborations 


\section{Introduction}

No fundamental symmetry explains the almost perfect conservation of charged Lepton Flavour, Lepton Number and Baryon Number quantum numbers in the Standard Model (SM). Even in the SM, Lepton Flavour Violation (LFV) and Lepton/Baryon Number Violation (LNV/BNV) are expected to occur at very high energies or densities [1]. The fact that neutrinos oscillate also enhances the SM cross-section for charged LFV and LNV processes but they still remain substantially below the experimental reach of detectors at any current or future collider.

Many New Physics (NP) models can enhance the lepton and baryon violating processes to a level that they could be detected by $e^{+} e^{-}$experiments such as BABAR or Belle. In Grand Unified Theories (GUTs), the quarks and leptons are no longer members of separate multiplets and the distinction between baryon and lepton number disappears. GUT theories can conserve or violate the difference in baryon and lepton number $(B-L)$ and this can alter the predictions for matter/antimatter asymmetry created through baryo- and lepto-genesis at the beginning of the Universe. NP models can introduce new particles such as additional Higgs bosons, particles that carry both lepton and baryon numbers, or models that simply introduce new couplings that break the assumption of equal strength couplings for the leptons.

With so many different NP models and such a large parameter space, it is important to make as many measurements as possible involving baryons, mesons, and leptons. BABAR and Belle collected approximately 470 and 770 million $B$-meson pairs at the $\Upsilon(4 S)$ resonance, respectively. As the luminosity-weighted $e^{+} e^{-} \rightarrow \tau^{+} \tau^{-}$cross-section is $\sim 0.92 \mathrm{nb}$, a similar number of $\tau^{+} \tau^{-}$ pairs were recorded. BABAR (Belle) went on to collect 121 (11) and 99 (158) million events at the $\Upsilon(3 S)$ and $\Upsilon(2 S)$ resonances, while Belle collected 102 and 7 million events at the $\Upsilon(1 S)$ and $\Upsilon(5 S)$ resonances, respectively. In addition, both experiments collected a large number of hadrons produced by the hadronization of the original quark pair produced by the $e^{+} e^{-}$annihilation; this dataset has not been exploited.

The analyses follow a similar pattern, with variations to take into account specific scenarios. In general, the energy difference $\Delta E=E_{B / \tau}^{*}-\sqrt{s} / 2$, where $E_{B / \tau}^{*}$ is the energy of the decaying particle in the centre-of-mass frame (CM) and $\sqrt{s}$ is the total energy of the $e^{+} e^{-}$system in the CM frame, and beam energy substituted mass $m_{\mathrm{ES}}=\sqrt{s / 4-p_{B / \tau}^{* 2}}$, where $p_{B / \tau}^{*}$ is the CM momentum of the decaying particle ${ }^{1}$, are combined with a multivariate discriminator using event-shape variables to separate the signal and background. The yields are then measured either by fitting the number of events using signal and background distribution functions or using background events outside a blinded signal region (SR) to estimate the expected yield in the SR and then counting events in the SR. This latter technique can be combined with simulation predictions for processes that are well understood.

For decays that involve neutrinos, the $e^{+} e^{-}$experiments can use the fact that the $B$-mesons and $\tau$-leptons are produced in pairs and decay back-to-back in the CM. One of the pair is fully reconstructed using hadronic or semileptonic decays. Although semileptonic decays still lose energy through the neutrino, the reconstructed $B$-meson or $\tau$-lepton can be used to impose energy and momentum constraints on the other member of the pair.

\footnotetext{
${ }^{1}$ The $B A B A R m_{\mathrm{ES}}$ definition is slightly different.
} 


\section{2. $\Upsilon(n S), B$-meson and $\tau$-lepton results}

CLEO placed 90\% CL branching fraction upper limits $B_{\mathrm{UL}}^{90 \%}$ on $\Upsilon(n S) \rightarrow \mu \tau$ of $\sim 10^{-5}$ that were already more stringent than theoretical predictions. BABAR looked at the $\ell^{+}\left(=\mu^{+}, e^{+}\right)$momentum endpoint to produce $B_{\mathrm{UL}}^{90 \%}\left(\Upsilon(2 / 3 S) \rightarrow \ell^{+} \tau^{-}\right)<(3.1-4.2) \times 10^{-6}$ [2]. Searches for rare and FCNC in decays such as $B \rightarrow h \ell^{+} \ell^{-}$and $B^{0} \rightarrow \ell^{+} \ell^{-}$are natural places to look for LFV as new particles can appear in the penguin loops. Measurements with $h=\pi, K, K^{*}$ and $\ell \ell=e \mu$ have produced $B_{\mathrm{UL}}^{90 \%}<(9.1-140) \times 10^{-8}$ [3]. If one of the leptons $\ell$ is allowed to be a $\tau$ then limits $B_{\mathrm{UL}}^{90 \%}<(3.0-7.5) \times 10^{-5}$ and $<(2.2-2.8) \times 10^{-5}$ for $B^{+} \rightarrow h^{ \pm} \tau \ell$ and $B^{0} \rightarrow \tau^{ \pm} \ell^{\mp}$, respectively [4], are reached.

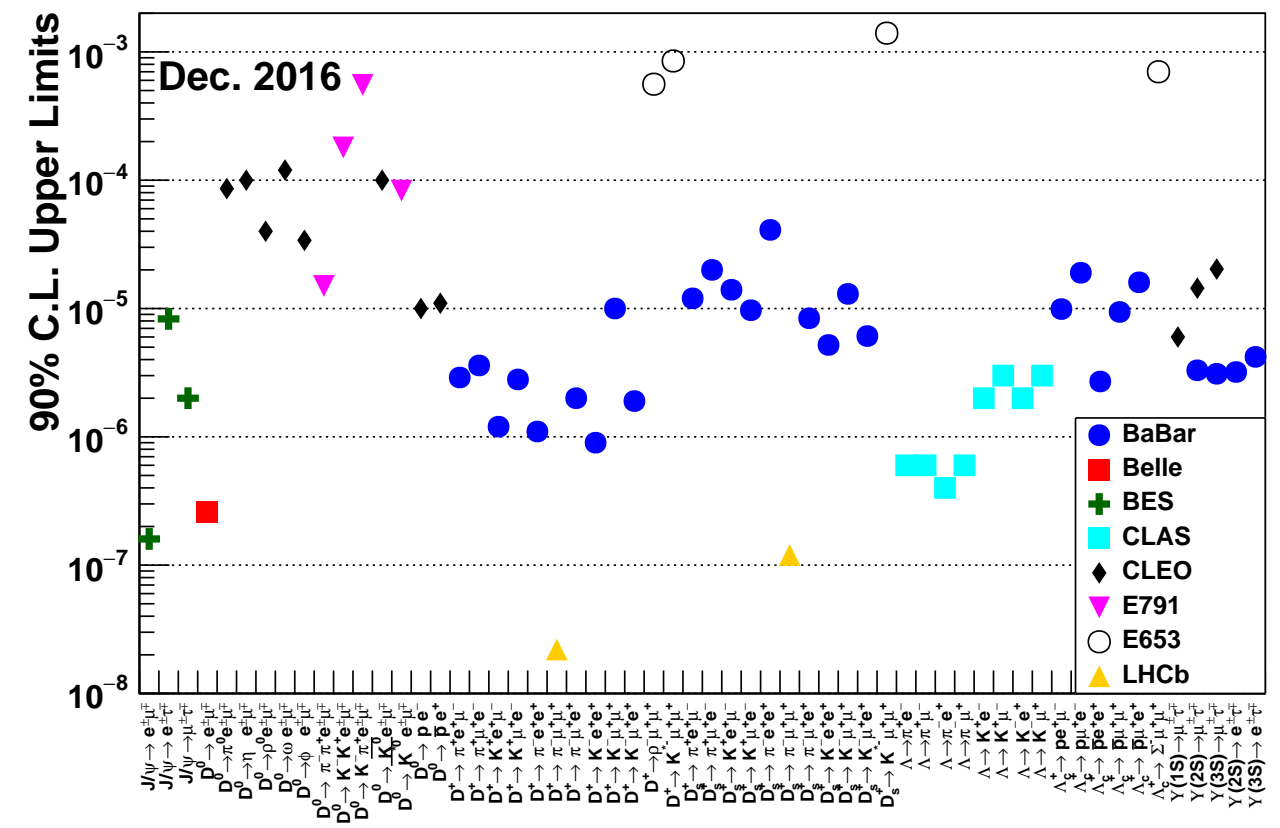

Figure 1: Summary of $\Upsilon$ and $\Lambda \mathrm{LNV/LFV} B_{\mathrm{UL}}^{90 \%}$ results reported here, together with other quarkonia results from other experiments [7].

A possible mechanism for the LNV decay $B^{+} \rightarrow X^{-} \ell^{+} \ell^{+}$is the exchange of a Majorana neutrino with a mass less than the $B$-meson mass but with an unknown coupling. Both Belle and BABAR have searched for the decay with $X^{-}=D^{-}, \pi^{-}, K^{-}, K^{*-}, \rho^{-}$and $\ell^{+} \ell^{+}=\mu^{+} \mu^{+}, e^{+} e^{+}, \mu^{+} e^{+}$. For $X^{-}=D^{-}, B_{\mathrm{UL}}^{90 \%}<(11-26) \times 10^{-7}$ while for the other $X^{-}, B_{\mathrm{UL}}^{90 \%}<(1.5-9.8) \times 10^{-7}$ [5]. The search for LNV and BNV has been performed in six decays with $B \rightarrow \Lambda_{(c)} \ell^{+}$, which are expected to be highly suppressed $\left(<10^{-29}\right)$. The decay $B^{-} \rightarrow \bar{\Lambda} \ell^{-}$also violates $B-L$ conservation. For the decays $B^{0} \rightarrow \Lambda_{c}^{+} \ell^{-}, B_{\mathrm{UL}}^{90 \%}<(180-520) \times 10^{-8}$, while for the four $B^{-} \rightarrow \Lambda \ell^{-}$decays $B_{\mathrm{UL}}^{90 \%}$ is in the range $(3.2-6.2) \times 10^{-8}$ [6]. Summaries of these results are shown in Figs. 1 and 2, together with results from other experiments, and with a number of other quarkonia and charmonium decays not discussed here [7].

Although usually interpreted in terms of the Type II Higgs Doublet models (2HDM), the discrepancy in the ratios of $B\left(B \rightarrow D^{(*)} \tau \nu_{\tau}\right)$ to $B\left(B \rightarrow D^{(*)} \ell v_{\tau}\right)$ can be interpreted in terms of different 


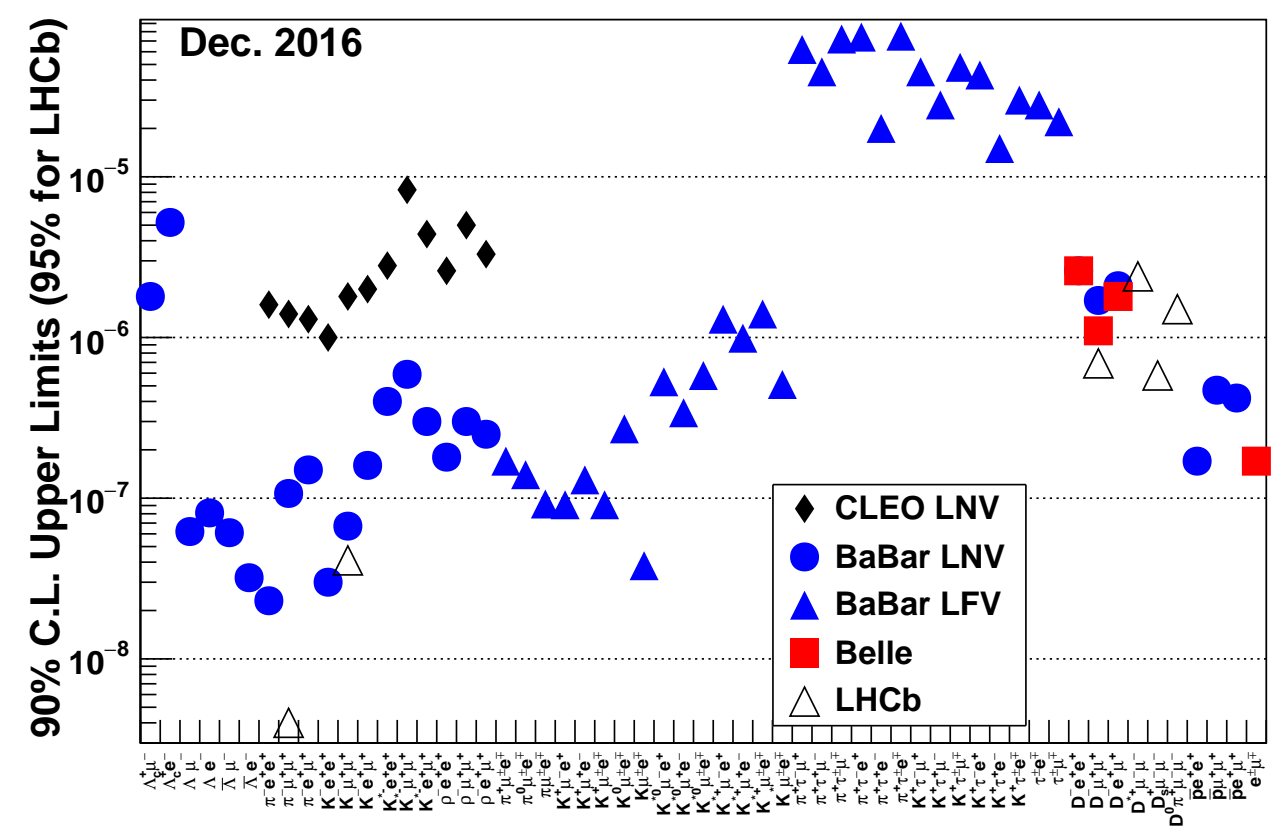

Figure 2: Summary of $B$-meson LNV and LFV $B_{\mathrm{UL}}^{90 \%}$ results for $B A B A R$ and Belle, together with earlier results from CLEO and $B_{\mathrm{UL}}^{95 \%}$ from $\mathrm{LHCb}[7]$.

couplings to the leptons and therefore a violation of Lepton Universality [8]. Measurements of the ratio of the neutrino-less branching fraction decays $B \rightarrow K \mu^{+} \mu^{-}$to $B \rightarrow K e^{+} e^{-}$at BABAR, Belle, and LHCb have shown discrepancies from the theoretical estimate of $1.0 \pm \sim 0.01$, with a combined result in the range $1<q^{2}<6 \mathrm{GeV}^{2} / c^{4}$ that is $2.6 \sigma$ from the SM prediction [9].

As shown in Fig. 3, the branching fraction upper limits on $\tau$ LFV and LNV are dominated by Belle, where full advantage has been taken of the large dataset and clean environment [10]. The measurements cover the range $B_{\mathrm{UL}}^{90 \%}<(1-4) \times 10^{-8}$ for most of the decays. In the absence of improved measurements of the lepton coupling constants $g_{\ell}$, the main recent development has been the reduction in uncertainty on the $\tau$ lifetime such that $\tau_{\tau}=(290.17 \pm 0.53 \pm 0.33) \times 10^{-15} \mathrm{~s}$. The branching fraction and lifetime are connected to the coupling constants through $B(\tau \rightarrow \ell v \bar{v}) / \tau_{\tau} \propto$ $g_{\tau}^{2} g_{\ell}^{2} m_{\tau}^{5} f\left(m_{\ell}^{2} / m_{\tau}^{2}\right) \delta_{W} \delta_{\gamma}$, where $f\left(m_{\ell}^{2} / m_{\tau}^{2}\right), \delta_{W}$, and $\delta_{\gamma}$ are correction factors. The current values of the coupling constant ratios from leptonic-processes only are $\left(g_{\tau} / g_{\mu}\right)=1.0010 \pm 0.0015$, $\left(g_{\tau} / g_{e}\right)=1.0029 \pm 0.0015$, and $\left(g_{\mu} / g_{e}\right)=1.0019 \pm 0.0014[10]$.

In conclusion, apart from LHCb's measurements with muons in the final state and some new results from BES and CLAS in quarkonia decays (not covered here), the last two years have been relatively quiet. This will change with new data from $\mathrm{LHCb}$, upgraded experiments such as MEG, and brand new experiments including Mu2e, Mu3e, and Belle 2. It is probable that the $B_{\mathrm{UL}}^{90 \%}$ reported here will improve by between one and two orders of magnitude over the next decade.

\section{References}

[1] F.R. Klinkhamer and N.S. Manton, Phys. Rev. D 30, 2212 (1984). 


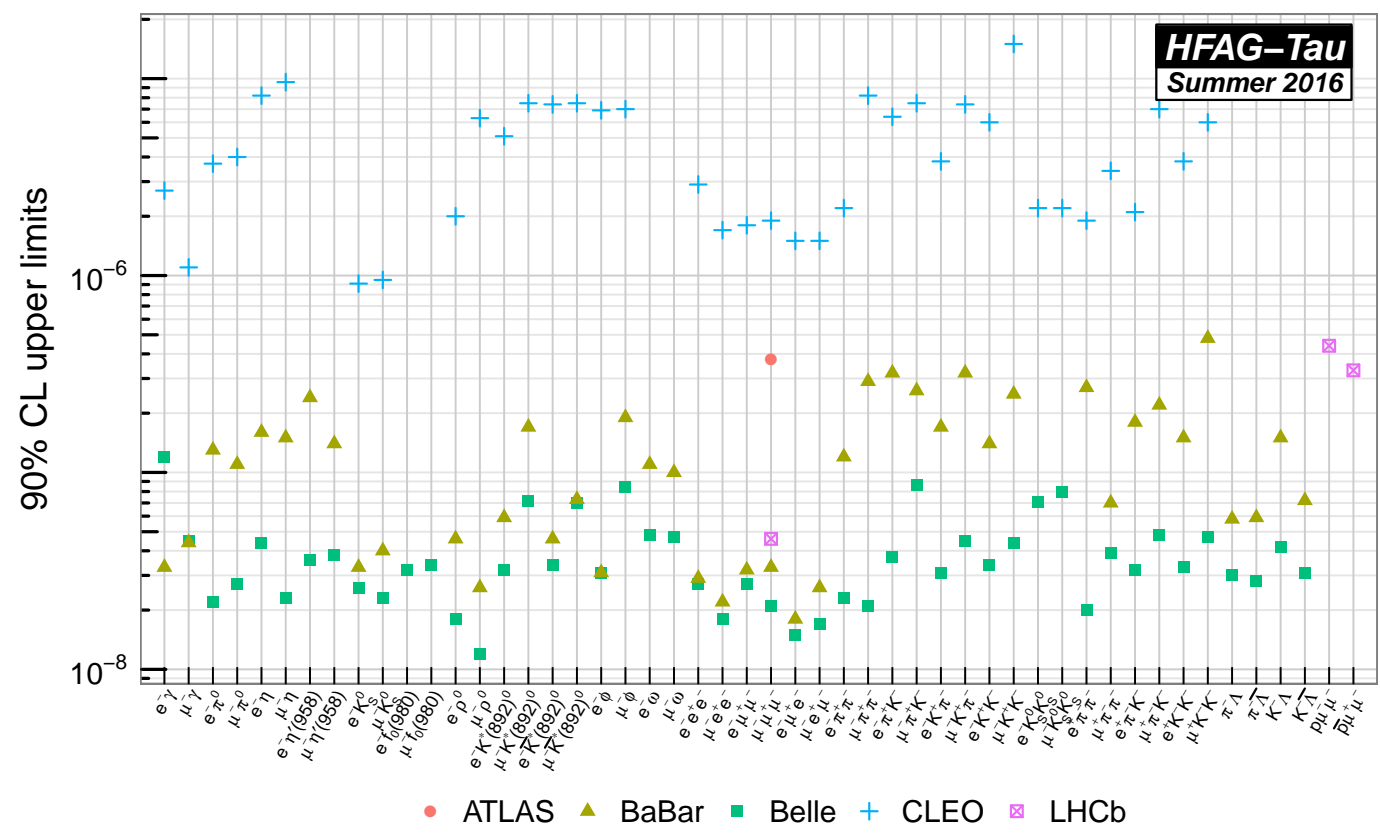

Figure 3: Summary plot of $B_{\mathrm{UL}}^{90 \%} \tau$-lepton decay LFV and LNV results [10].

[2] W. Love et. al., (CLEO Collaboration), Phys. Rev. Lett. 101, 201601 (2008); J.P. Lees et. al., (BABAR Collaboration), Phys. Rev. Lett. 104, 151802 (2010).

[3] B. Aubert et. al., (BABAR Collaboration), Phys. Rev. D 77, 032007 (2008); B. Aubert et. al., (BABAR Collaboration), Phys. Rev. D 77, 091104 (2008); B. Aubert et. al., (BABAR Collaboration), Phys. Rev. D 73, 092001 (2006); J.P. Lees et. al., (BABAR Collaboration), Phys. Rev. D 85, 071103 (2012); B. Aubert et. al., (BABAR Collaboration), Phys. Rev. Lett. 99, 051801 (2007).

[4] J.P. Lees et. al., (BABAR Collaboration), Phys. Rev. D 86, 012004 (2012); B. Aubert et. al., (BABAR Collaboration), Phys. Rev. D 77, 091104 (2008).

[5] J.P. Lees et. al., (BABAR Collaboration), Phys. Rev. D 89, 011102 (2014); J.P. Lees et. al., (BABAR Collaboration), Phys. Rev. D 85, 071103 (2012); O. Seon et. al., (Belle Collaboration), Phys. Rev. D 84, 071106 (2011).

[6] P. del Amo Sanchez et. al., (BABAR Collaboration), Phys. Rev. D 83, 091101 (2011).

[7] Particle Data Group, C. Patrignani et. al., Chin. Phys. C40 (2016) 100001.

[8] Y. Sato et. al., (Belle Collaboration), Phys. Rev. D 94, 072007 (2016); M. Huschle et. al., (Belle Collaboration), Phys. Rev. D 92, 072014 (2015); J.P. Lees et. al., (BABAR Collaboration), Phys. Rev. D 88, 072012 (2013); J.P. Lees et. al., (BABAR Collaboration), Phys. Rev. Lett. 109, 101802 (2012).

[9] J.P. Lees et. al., (BABAR Collaboration), Phys. Rev. D 86, 032012 (2012); J.-T. Wei et. al., (Belle Collaboration), Phys. Rev. Lett. 103, 171801 (2009).

[10] Y. Amhis et. al., (Heavy Flavor Averaging Group), arXiv:1612.07233.

[11] K. Belous et. al., (Belle Collaboration), Phys. Rev. Lett. 112, 031801 (2014). 